\title{
MODELO DE COMPETENCIAS PROFESIONALES ESPECÍFICAS PARA LA FORMACIÓN DE LOS ESPECIALISTAS EN MEDICINA GENERAL INTEGRAL
}

\author{
SPECIFIC PROFESSIONAL COMPETENCES MODEL FOR THE \\ TRAINING OF SPECIALISTS IN GENERAL INTEGRAL MEDICINE
}

\author{
Rosalina Ramos Hernández ${ }^{1}$ \\ Alejandro Antuan Díaz Díaz ${ }^{1}$ \\ Norberto Valcárcel Izquierdo \\ ${ }^{1}$ Facultad de Ciencias Médicas “Enrique Cabrera”. La Habana. Cuba. \\ 2 Universidad de Ciencias Pedagógicas "Enrique José Varona". La Habana, Cuba. \\ rosalinara@infomed.sld.cu
}

\section{RESUMEN}

La formación por competencias en la educación de postgrado, se revela desde la connotación mundial relacionada con la calidad de la preparación de los profesionales del sector de la salud. En la formación del médico general integral; definir las competencias profesionales específicas a desarrollar, es importante por su relación con la calidad del desempeño profesional de este especialista y de la atención médica que brinda a la población, con el objetivo de diseñar un modelo de competencias profesionales específicas para la formación del especialista en Medicina General Integral (MGI), que mejore su desempeño profesional, se realizó una investigación de desarrollo tecnológico, a través de la sistematización, el análisis documental, el estudio histórico lógico y la modelación realizada, se elaboró un Modelo de competencias profesionales específicas a desarrollar en la formación del especialista en Medicina General Integral identificadas como comunicativa; clínica; epidemiológica; en educación en salud; docente; investigativa y gerencial que posibilitan mejoría en el desempeño profesional y humano de estos especialistas. La investigación enriquece a las Ciencias Pedagógicas, en general, y a la Teoría de la Educación Avanzada, en particular, con la identificación de regularidades que se establecen en las relaciones entre la organización académica de los programas de especialidades a partir de la adquisición y desarrollo de las competencias profesionales específicas y el desempeño profesional de los estudiantes de postgrado, que favorece la integración entre la Universidad y la Sociedad, desde una concepción desarrolladora y humanista que involucra al individuo con la formación que realiza de forma activa.

Palabras clave: formación por competencias; competencias profesionales específicas; recursos humanos en formación; desempeño profesional; modos de actuación profesional.

\begin{abstract}
The formation for competitions in the graduates degree education is revealed from the world connotation related with the quality of the preparation of the professionals of the sector of the health. In the general integral practitioner's formation; to define the professional specific competitions to develop, it is important for their relationship with the quality of this specialist's professional acting and of the medical care that offers to the population, with the objective of proposing the professional specific competitions for the specialist's formation in Integral General Medicine (MGI) that improves their professional acting, was carried out an investigation of technological development, being systematized the work of several authors about this topic; through the historical-logical study and the documental carried out analysis, to Model of professional specific competitions was elaborated to develop in the specialist's formation in Integral General Medicine identified ace talkative; clinic; epidemic; in education in health; educational; investigative and managerial that facilitates you improvement in the professional and human acting of these specialists. The investigation enriches to the Pedagogic Sciences, in general, and to the Theory of the Advanced Education, in matter, with the identification of regularities that they settle down in the relationships among the academic organization of the programs of specialties starting from the acquisition and development of the professional specific competitions and the professional acting of the
\end{abstract}


graduates degree students that it favors the integration between the University and the Society, from to development and humanist conception that involves the individual with the formation that has carries out in an activates way.

Keywords: formation for competitions; professional specific competitions; human resources in formation; I carry out professional; ways of professional performance.

Recibido: 06/11/2017; $\quad$ Aceptado: 11/12/2017; $\quad$ Publicado: 19/12/2017

\section{Introducción}

El enfoque de formación basado en competencias se erige como uno de los caminos para acercarse al logro de la calidad deseada en los egresados universitarios. Este enfoque, se sustenta en la sistematización relacionada con los razonamientos y deducciones emanadas de los estudiosos a nivel internacional y nacional. ${ }^{(1)}$

Según establece el Reglamento de la Educación de Posgrado de la República de Cuba (2), la formación académica dentro de la educación de postgrado tiene como objetivo la formación de profesionales competentes, capaces de resolver los problemas de la sociedad a través de la investigación e innovación.

En la sistematización realizada por la autora se pudo establecer que a partir de la Cumbre Mundial de Educación Médica celebrada en Edimburgo en 1993, se desarrolla un movimiento internacional para la búsqueda de un cambio articulado entre la educación médica, la práctica médica y la organización de salud, que posibilite la formación de un profesional que responda a las necesidades económico sociales de sus respectivos países, pero a la vez sea capaz de enfrentar los retos científicos y tecnológicos que exige la presente centuria, sobre la base de una cooperación e interrelación tanto en los ámbitos nacionales como internacionales.

En el análisis documental realizado por la autora a los documentos normativos de la formación de especialista en Medicina General Integral, en particular al Plan de Estudio de la Residencia de Medicina General Integral se identifica que: "El médico general integral es un especialista de amplio perfil capaz de garantizar la atención a la población asignada sin distingos de edad o género, con un enfoque integrador de los aspectos biológicos, sociales, psíquicos y ambientales, con acciones de promoción, prevención, diagnóstico, tratamiento y rehabilitación." (3)

Sin embargo en el diseño del plan de estudio no se aprecia en ninguna de las versiones una integración de los contenidos y habilidades practicas a formar en el residente, que le permita alcanzar un amplio perfil que le facilite el garantizar la atención a la población.

La autora desde resultados previos, derivados de investigaciones propias relacionadas con la evaluación de las habilidades de los recursos humanos en la formación como especialistas en Medicina General Integral(4), determina la existencia de la necesidad de definir cuáles son las competencias profesionales específicas de la especialidad que realmente debe desarrollar el recurso humano en formación, ya que la inexistencia de la mismas conlleva a la repetición innecesaria de contenidos del pregrado sin la profundización adecuada para el nivel de especialización que se exige a través del modelo del egresado y que se manifiesta en un desempeño profesional no acorde a las dinámicas sociales y económicas actuales.

Otros investigadores en el contexto de la educación médica superior del ámbito nacional, relacionados con la formación de recursos humanos vinculados a la especialidad en Medicina General Integral, son los doctores en ciencias, Dr. C. Alemañy Pérez (2008) (5), aborda el cumplimiento del modelo del egresado como especialista en Medicina General Integral en los recursos humanos en formación; el Dr. C Díaz (2012) ${ }^{(6)}$ estudia la evaluación de los procesos formativos en los que se sustenta la formación de recursos humanos en la especialidad Medicina General Integral; el Dr. C Salas (2012) (7) estudia la formación de competencias profesionales y su evaluación a través del desempeño profesional en las Ciencias Médicas.

\section{Objetivo}

Diseñar un modelo de competencias profesionales específicas para la formación del especialista en Medicina General Integral.

\section{Material y métodos}

Se realizó una investigación de desarrollo tecnológico, del sistema diseñado para la 
formación de los recursos humanos en la especialidad de Medicina General Integral, para proponer las competencias profesionales específicas para la formación de los especialistas en Medicina General Integral, que permita el mejoramiento de su desempeño profesional para lo cual se utilizaron los métodos teóricos:

Análisis documental: para valorar la planificación curricular de la residencia médica en Medicina General Integral, desde la organización del perfil y sus nexos con la orientación metodológica que se realizan a los docentes. Se analizaron los siguientes documentos, a través de una guía documental:

1. Ministerio de Educación Superior. Resolución Ministerial No. 132/2004. Reglamento de la Educación de Posgrado de la República de Cuba.

2. Ministerio de Salud Púbica. Plan de Estudios de la Residencia de MGI. (1ª versión. 1985; 2a . versión1990; 3a. versión1999- 2000; 4ta. Versión 2004)La Habana: MINSAP;

Histórico - Lógico: posibilitó el estudio del desarrollo histórico de la formación de especialistas en Medicina General Integral, lo que permite aportar tendencias en la formación de los residentes de Medicina General Integral en la actualidad, base para la construcción de sus competencias profesionales específicas. Este enfoque se integró en el análisis documental de las 4 versiones del programa de formación de este especialista

Sistematización: se empleó en la identificación y conceptualización de la formación de especialistas, el desempeño profesional y las competencias profesionales específicas para la construcción del marco teórico relacionado al objeto y campo de acción. Se realizó la sistematización de los resultados de investigadores del tema dentro de las Ciencias Pedagógicas y de la Educación Médica Superior.

Modelación: Se empleó para diseñar el Modelo de Competencias Profesionales Específicas para la formación de los especialistas en Medicina General Integral.

\section{Resultados}

En la base de la investigación con carácter científico es necesario realizar determinadas construcciones teóricas, que constituyen diseños abstractos que revelan las cualidades del objeto de estudio, y se identifica en esta investigación, como: la formación de especialista en Medicina General Integral, así como las relaciones existentes entre sus componentes, las competencias profesionales específicas como proceso y la valoración de los sustentos teóricos de las Ciencias Pedagógicas que sirven de guía para su elaboración. Por ese motivo, la autora necesitó profundizar en los fundamentos acerca de los modelos como resultado científico y la modelación como método de investigación.

Para el proceso de modelación de la formación de competencias profesionales específicas de los especialistas en Medicina General Integral, se realizó la sistematización de las investigaciones de varios autores, en particular los doctores Pérez, en 2006 (8) y Añorga, en $2014{ }^{(9)}$ que coinciden en la identificación de un grupo de características comunes en los modelos de investigación:

1. Constituyen una reproducción que esquematiza las características de la realidad, permitiendo adentrarse en su estudio. El modelo debe cumplir con determinado nivel de analogía estructural y funcional con la realidad de manera que permita extrapolar los datos obtenidos en el modelo sobre el objeto o fenómeno estudiado.

El Modelo de Competencias Profesionales Específicas para la formación de los especialistas en Medicina General Integral expresa las relaciones entre los componentes de la formación por competencias profesionales específicas del especialista en Medicina General Integral, desde los componentes del diseño curricular de las residencias médicas (Académico, Laboral e Investigativo), el micro currículo (programas de los módulos y áreas de actuación del desempeño profesional en las rotaciones que realiza), se reproduce la realidad en la formación del estudiante y profundiza en este proceso de formación cuando al identificar las competencias profesionales específicas que deben adquirir los egresados para desempeñarse con eficiencia en el mundo laboral.

2. Debe ser operativo y más fácil de estudiar que el fenómeno real. Se puede modificar, transformar, someter a estímulos diversos con vista a su estudio.

El Modelo de Competencias Profesionales Específicas para la formación de los especialistas en Medicina General Integral, se asiste de una metodología que permite a los profesores y tutores de la facultad de Ciencias Médicas dirigir la formación de competencias profesionales específicas desde el proceso de formación de los especialistas en Medicina General Integral, acercándolos, a las 
exigencias de la sociedad y a las necesidades de la comunidad en la que laboran.

Un fenómeno de la realidad puede ser encarnado por varios modelos y viceversa, en un mismo modelo pueden encarnarse varios fenómenos, en esta investigación, coligado con la formación de competencias de los especialistas en Medicina General Integral, confluyen las valoraciones de las diferentes áreas de actuación del desempeño profesional del residente de la especialidad en Medicina General Integral, vinculadas con el componente académico, con el laboral, con el docente y con el investigativo, que en su integración miden la formación profesional de estos.

En la etapa de exploración de la investigación, y como resultado de la sistematización realizada en la misma, la autora considera diversas alternativas que satisfacen el proceso de formación de competencias profesionales específicas de los especialistas en Medicina General Integral, como propuestas se identificaron: los sistemas de competencias definidas en el programa, el desarrollo de la evaluación de las competencias, definidas en el modelo del egresado, en los residentes, por los profesores.

Se construye un Modelo de Competencias Profesionales Específicas para la formación de los especialistas en Medicina General Integral, ya que la autora reconoce la posibilidad de representar otras modelaciones, tales como: el modelo de competencias profesionales específicas en los especialistas en Medicina General Integral, las relaciones dialécticas que se manifiestan en el proceso de formación académica o la relación que se establece entre la calidad del proceso de formación y la transformación del residente como individuo, en la medida en que la adquisición de conocimientos, habilidades y valores posibiliten el mejoramiento del desempeño profesional del residente sobre la población que atiende.

3. Las variables, relaciones y constantes del modelo se interpretan a partir de una teoría científica.

La constante en este modelo se sustenta en la adquisición de competencias profesionales específicas por parte de los residentes, en su formación como especialista de Medicina General Integral, al garantizar por parte de los profesores, la actualización de los contenidos propios de su desempeño profesional, favoreciendo el desarrollo de las funciones declaradas en el modelo del egresado, según el plan de estudio del año 2004. (10)
Los modelos se caracterizan generalmente por su provisionalidad, su adaptabilidad, su optimización, su carácter organizador en el proceso, su utilidad teórica, investigativa, tecnológica y práctica.

Para lograr la utilización de la modelación, como método y el Modelo de Competencias Profesionales Específicas para la formación de los especialistas en Medicina General Integral, como resultado científico, se busca estructurar las dimensiones, componentes, criterios e indicadores para el cumplimiento del modelo del egresado, entre otras fuentes, la autora desarrolla su modelo de competencias basado en los tres perfiles determinados por el programa formativo de la residencia en Medicina General Integral (versión del 2004): el político-ideológico, el profesional y el ocupacional para abordar las exigencias socioculturales de este egresado y las necesidades en materia de salud de la población que atiende, el análisis realizado al objeto de estudio y la síntesis del mismo, le brinda a este modelo, un carácter flexible, que le brinda la capacidad de adaptarse a nuevos cambios y ser particularizado en dependencia del escenario donde sea aplicado.

En la tesis del doctor Bringas Linares en 1999 (11) se proponen principios en los que se debe sustentar todo modelo. La autora considera posible valorar cómo se manifiestan estos principios en el Modelo de Competencias Profesionales Específicas para la formación de los especialistas en Medicina General Integral que se propone:

- Principio del enfoque sistémico. Posibilita revelar las cualidades resultantes del objeto de investigación, mediante las relaciones que se tienen que dar entre los componentes el Modelo de Competencias Profesionales Específicas para la formación de los especialistas de la especialidad en Medicina General Integral.

La primera relación se da entre el micro currículo y el macro - currículo definido por el programa de la especialidad en Medicina General Integral, esta relación se expresa en los procesos que ocurren en el componente académico, laboral e investigativo y que en su integración muestran las competencias profesionales específicas a desarrollar en los residentes.

La segunda relación se manifiesta en el cumplimiento del modelo del egresado diseñado para esta formación profesional y los modos de actuación declarados en su desempeño profesional, lo que favorece la calidad de los servicios que se brindan por 
estos profesionales en el nivel de atención primaria de salud.

Además en este Modelo de Competencias Profesionales Específicas se establece otra relación, entre los problemas identificados en el actual proceso de formación de los residentes en Medicina General Integral y la formación por competencias profesionales específicas de los especialistas y las formas evaluativas que se identifican en cada componente del diseño curricular de la residencia médica, pero con insistencia en el proceso de auto-evaluación dela adquisición de competencias que demuestra el carácter consciente del proceso de formación y la autonomía para establecer las estrategias de aprendizaje y de auto-ayuda de cada individuo en la formación desde el Modelo de Competencias Profesionales Específicas que se propone.

- Principio de la simplicidad y la asequibilidad. Sin perder el valor y el carácter científico, el modelo debe ser comprensible, funcional y operativo.

El Modelo de Competencias Profesionales Específicas que se propone; para su aplicación práctica, se asiste de una metodología que indica su implementación por los directivos y profesores de la facultad que conducen el proceso de formación de los especialistas en Medicina General Integral, para lograr la adquisición de las competencias profesionales específicas identificadas, que se corresponden con las funciones declaradas en el perfil del egresado, ajustadas al escenario en el que se forma y desempeña, a partir del principio de educación en el trabajo que se aplica en el proceso docente educativo dentro de la educación médica en Cuba, al comprobar el grado de transformación del residente en su desempeño profesional y humano que se logra en el proceso de formación del mismo.

- Principio de la consistencia lógica del modelo. Asegura estabilidad, solidez y fundamentación científica a los elementos teóricos que sustentan el modelo.

El Modelo de Competencias Profesionales Específicas para la formación de los especialistas de Medicina General Integral, se fundamenta teóricamente en las Ciencias Pedagógicas, en general, y en la identificación de las regularidades en la organización académica de los programas de especialidades a partir de las formación por competencias profesionales específicas y el desempeño profesional de los estudiantes de la educación de postgrado, en particular; lo cual favorece la transformación del proceso de atención primaria en salud de la población en la que se desempeñan estos profesionales en formación.

- Principio de deducción por analogía. Mediante el mismo se pretende establecer semejanzas entre ciertas facetas, cualidades y componentes del objeto real y el modelado.

El propósito de esta investigación es transformar la formación basada en la adquisición de competencias profesionales específicas y el mejoramiento del desempeño profesional y humano de los residentes en Medicina General Integral, por lo cual, se manifiestan etapas propias de las investigaciones sociológicas, entre ellas, las vinculadas a las Ciencias Pedagógicas como: etapa exploratoria, etapa de construcción del marco teórico, etapa de diagnóstico o trabajo empírico, etapa de construcción de la propuesta de solución y etapa de comprobación, constatación o validación en la práctica de los cambios que ocurren con la aplicación del Modelo de Competencias Profesionales Específicas para la formación de los especialistas en Medicina General Integral.

Un modelo constituye una construcción teórica que pretende informar (explicar un fragmento acotado de la realidad). Entre sus aspectos importantes a destacar sobre los modelos de competencias se pueden enunciar:

- No sólo se pretende describir y explicar la realidad, sino informar como intervenir en ella para transformarla.

- Se fundamenta en un conjunto de aportes de otras disciplinas y áreas del saber: filosofía, psicología, epistemología, así como del mundo de los valores, ideologías y cosmovisiones.

- Constituyen teorías sobre los procesos formativos.

En el estudio realizado a los criterios aportados por Pérez y Añorga y a los principios planteados por Bringas, en los que se sustenta el Modelo de Competencias Profesionales Específicas, la autora identifica relaciones que fundamentan el proceso de modelación pedagógica.

\section{Dimensión Filosófica}

Mediante el estudio de los fundamentos de las concepciones pedagógicas que sustentan el proceso de formación por competencias profesionales específicas de los residentes en Ciencias Médicas, en especial en Medicina General Integral, se detecta que constituye una manera para satisfacer las necesidades de los residentes en Medicina General Integral en su 
formación profesional.

En opinión de la autora, la concepción filosófica se expresa, en la relación existente entre la actividad asistencial que ejercen los residentes de Medicina General Integral y el grado de transformación del estado de salud de la población que atiende, lo que se favorece al identificar sus modos de actuación.

Los cambios sucedidos en el sistema de salud en la actualidad, con vista de la mejoría en la calidad de la atención en salud, lo que provoca que el proceso de formación de los residentes en Medicina General Integral haya sufrido transformaciones que se ejecutan fundamentalmente, a nivel de los escenarios docentes, al crear las condiciones favorables 0 desfavorables alrededor de la formación de estos recursos humanos.

Al aceptar la práctica como criterio de la verdad y asumir los aspectos positivos de desempeño profesional de estos profesionales de la salud, se hace posible precisar las posiciones epistemológicas y éticas del Modelo de Competencias Profesionales Específicas propuesto. Es indudable que el modelo de competencias profesionales específicas para la formación de los residentes en Medicina General Integral propuesto depende de su desempeño profesional y la pertinencia de los conocimientos, habilidades $y$ valores $y$ actitudes que reciben y refuerzan, en la residencia médica, basándose el mismo en aquellas acciones dirigidas a establecer el criterio de la verdad sobre estos acápites, que deben revertir en el mejoramiento del desempeño profesional de estos recursos humanos.

En los fundamentos del modelo de competencias profesionales específicas para la formación de los especialistas en Medicina General Integral se demuestran los principios de la filosofía de la educación (12), tales como: el carácter masivo y equidad, la combinación estudio y trabajo con la participación democrática, abierta a la diversidad. Se hace explícito la relación con la cultura, la formación, la identidad y la transformación del entorno para la formación integral de los recursos humanos especializados del sector de la salud.

\section{Dimensión Pedagógica}

El Modelo de Competencias Profesionales Específicas propuesto se cimienta en los referentes teóricos de las ciencias pedagógicas. En la sistematización, realizada a la obra de los autores: Añorga (13), Freire (14), Pérez (15), Ferrer (16), Pérez (17), Torres (18) Martín (19), Moreno(20), Valiente y Guerra(21), entre otros investigadores del tema, se encontró que la adquisición de competencias profesionales específicas en los procesos de formación ha evolucionado en ascenso como consecuencia directa e indirecta de la propia práctica y su trascendencia al ámbito internacional por la formación de recursos humanos especializados en Medicina General Integral, en el mundo.

Todo lo cual permite una mayor comprensión en su abordaje, en tanto docentes, directivos, residentes y usuarios (pacientes y administradores en salud) que se vincula a la formación por competencias profesionales específicas de los residentes en Medicina General Integral; concuerdan en reconocer como forma del postgrado académico, su capacidad de abarcar a todas los componentes objetos de la competencias (componentes personales del proceso formativo) y a los usuarios de sus resultados (pacientes).

La Dra. Añorga en su obra Modelos de competencias plantea que:

Para organizar sistemas o procesos de formación por competencias, se deben considerar un conjunto de condiciones que garantizan un resultado favorable a la formación, estas son las siguientes:

1. Las competencias deben ser identificadas a partir de un proceso de diagnóstico.

2. Las competencias que se quieren formar deben manifestar sus relaciones con los objetos de la profesión o el desempeño.

3. Las competencias que se desean modelar deben verificarse ante especialistas de la institución empleadora, expertos del área de conocimientos o consensuadas con los propios estudiantes que serán formados y que cuentan con las vivencias que la práctica cotidiana les aporta.

4. Cada competencia modelada debe identificar las formas de evaluación que permitirá constatar el grado de adquisición de forma permanente y sistémica, en su relación con los servicios que realiza y en correspondencia con el resto de las que ya posee 0 que se le están formando simultáneamente.

5. La evaluación de las competencias debe tomar en consideración los conocimientos, las actitudes, los valores propios del desempeño y los modos de actuación que en el desempeño requiere el sujeto, como las principales fuentes de evidencia $y$ criterios para la medición de su obtención.

6. La formación por competencias es un 
proceso de aprendizaje personalizado e individualizado, en tanto que no debe estar sujeto a rígidas estrategias o dosificaciones que avancen el programa sin la constancia de que ya poseen los conocimientos, habilidades, valores, actitudes y modos de actuación necesarios para continuar la formación hacia un nivel mayor de desarrollo.

7. La formación por competencias requiere de una retroalimentación constante del docente que las forma, los materiales principales empleados como medios de enseñanza deben ser aquellos que reflejen situaciones reales y experiencias en el trabajo en sus diferentes objetos o áreas de desempeño.

8. La formación por competencias debe estar dirigida en su mayoría a la adquisición de experiencias prácticas, sin olvidar los temas 0 áreas del conocimiento que fundamentan sus acciones. Estas pueden estar relacionadas con hechos, conceptos, principios y otro tipo de conocimiento deben ser parte integrante de las tareas y funciones propias de puesto de trabajo o cargo que ocupen (Perfil ocupacional). (22). Desde esta concepción se manifiesta la relación entre la formación por competencias profesionales específicas y el mejoramiento del desempeño profesional de los residentes de Medicina General Integral que repercute en la integración entre la Universidad y la Sociedad, involucrando al individuo con la formación que realiza de forma activa y mejorando a su vez, la calidad en el servicio de salud que brinda.

La sistematización realizada a los procesos de formación, educación de postgrado y competencias, llevada al contexto de la especialidad de los residentes de la salud, le permitieron a la autora definir operacionalmente el Modelo de Competencias Profesionales Específicas para la formación de los especialistas en Medicina General Integral como: proceso pedagógico profesional permanente que comienza en la formación básica y continúa en la especializada, que provoca un cambio continuo en todos los niveles de desempeño profesional y humano, a través del cual se logra alcanzar la eficiencia en la adquisición y/o desarrollo de las competencias profesionales específicas exigidas por el modelo profesional y donde se manifiestan las dos dimensiones: la técnica profesional y la humana; se realiza con el objetivo de elevar los conocimientos científicos en una rama del saber, así como las habilidades y valores vinculadas con estos.

La valoración de la formación que reciben en la especialidad de postgrado, donde se encuentra la residencia en Medicina General Integral, se refleja en sus modos de actuación, perfecciona su desempeño profesional y logra su mejoramiento profesional y humano, para devolverlo a la sociedad como un individuo mejor preparado, capaz de satisfacer las necesidades siempre crecientes de la sociedad, a partir del reconocimiento en la atención médica a la comunidad.

Dada su vinculación con la formación de los residentes en Medicina General Integral, otro de los fundamentos pedagógicos en los que se sustenta el Modelo de Competencias Profesionales Específicas propuesto lo constituyó la teoría de la Educación Avanzada.

La teoría de la Educación Avanzada constituye el pilar fundamental en el que se sustenta esta investigación porque se profundiza en su objeto de estudio, sus relaciones esenciales y el Modelo de Competencias Profesionales Específicas que se propone pretende enriquecerla, al indagar en otras vertientes de la misma y a su consolidación como paradigma educativo alternativo dentro del contexto cubano e iberoamericano. Esta teoría ofrece un sistema de relaciones, regularidades, principios y leyes, que le brindan coherencia lógica interna a los procesos, fenómenos y sujetos que estudia para lograr el mejoramiento profesional y humano.

El Modelo de Competencias Profesionales Específicas que se propone es expresión del grado de madurez y desarrollo propio que posee esta teoría, ya que las acciones que propone pueden aplicarse a otras modalidades formativas dentro de la educación de postgrado ya sea en las ciencias médicas, como fuera de estas.

El Modelo de Competencias Profesionales Específicas para la formación de los especialistas en Medicina General Integral modelado pedagógicamente constituye parte de la realidad que rodea la formación de estos sujetos, las relaciones entre los participantes, sujetos y el objeto del proceso de formación y responde a una necesidad histórico concreta.

El redimensionamiento del sistema de salud en la actualidad exige la construcción de modelos de competencias que, desde una visión renovadora, respondan a las necesidades de los profesionales de la medicina y las exigencias y demandas de salud actual, tanto en el ámbito nacional como internacional. Con respecto a ello la doctora Terrero destaca que "cada época en correspondencia con el nivel de desarrollo de sus fuerzas productivas exige de determinado tipo de hombre para cada lugar concreto. De esta dinámica interna emanan las 
formas concretas del ser social, psicología social y todos sus productos, donde tienen lugar las teorías pedagógicas y sus modelos." (23) Por ello, como reflejo que son de las condiciones materiales de vida de los hombres, tienen un carácter histórico - social.

El Modelo de Competencias Profesionales Específicas, nace como una necesidad objetiva de una época y de una sociedad en constante transformación, lo que resulta una posible solución al problema de la formación por competencias profesionales específicas de los especialistas en Medicina General Integral, constituyendo así, la proyección de una realidad futura e ideal a alcanzar; al aportar una innovadora vía para la formación por competencias profesionales específicas, ya que le permite al residente comportarse como un agente activo en su proceso de formación y valora de una forma dinámica su desempeño profesional.

En el plano social el Modelo de Competencias Profesionales Específicas pretende preparar a los docentes y residentes, de la formación de especialistas en Medicina General Integral en Boyeros, para que sean capaces de satisfacer las demandas de la sociedad cubana actual a través del mejoramiento de su desempeño profesional, al utilizar como vía el proceso de formación, transformándose tanto la calidad humana y profesional de los residentes y docentes, como el estado de salud de la población que atienden.

\section{Dimensión Psicológica}

En la Educación Superior es muy importante el papel que desempeña el recurso humano en formación, la actitud a ser formado contribuye con la modificación, perfeccionamiento y transformación del sujeto durante la formación profesional, por lo que pueden participar de una forma más activa y consciente en los procesos de formación, a partir de las rotaciones, actuaciones y desempeños que se logran desde el currículo de la residencia médica, a ello se incluye la relación existente entre la auto-preparación, la auto-evaluación y la adquisición de las competencias profesionales específicas como proceso de formación, concebido como un proceso desarrollador que aporta elementos enriquecedores a la formación del sujeto.

El residente en Medicina General Integral se comporta ante su proceso de formación, como un sujeto capaz de tomar decisiones en relación con su vida profesional y personal, trasladando hacia su desempeño profesional elementos novedosos, validados por la práctica como criterio de la verdad y que los profesores deben monitorizar, para corregir tendencias negativas o para asegurar la satisfacción de nuevas necesidades de aprendizajes en estos sujetos. Se debe garantizar en la organización de los procesos de formación, la existencia de espacios o métodos activos que favorezcan la transformación del residente, tanto en lo profesional, como en lo personal, con un comportamiento activo, crítico, reflexivo, productivo, comunicativo y colaborador, que favorezca: el mejoramiento profesional y humano del residente y la calidad del estado de salud de la población.

Las características individuales de cada residente deben ser tomadas en consideración por los docentes que intervienen en el proceso de formación, para la adecuada planificación, ejecución y desarrollo de la adquisición de competencias profesionales específicas de los mismos. Se debe tener en cuenta por los profesores que las metodologías diseñadas para el desarrollo de competencias profesionales específicas en el proceso de formación, garanticen una participación activa de los residentes.

Los residentes en su gran mayoría son jóvenes, etapa en la que se alcanza una mayor estabilidad en torno a los motivos, intereses, puntos de vista propios, lo que hace que los residentes sean más conscientes de su propia experiencia y de las de los que los circundan, lo que favorece la formación de convicciones morales y el perfeccionamiento de su personalidad.

\section{Dimensión Ético Legal}

El Modelo de Competencias Profesionales Específicas propuesto contribuye a la formación axiológica de los recursos humanos que se vinculan al mismo desde su carácter universal y en lo particular se evidencia en los modos de actuación capaces de transformar la sociedad en virtud del sistema legal imperante, además posee carácter longitudinal y transversal de la formación y ve su máxima expresión en el desempeño profesional.

\subsection{Estructura del Modelo de Competencias Profesionales Específicas para la formación de los especialistas en Medicina General Integral}

El Modelo de Competencias Profesionales Específicas para la formación de los especialistas en Medicina General Integral se estructura a partir de los presupuestos identificados por la doctora De Armas Ramírez y otros (2004) (24), con los siguientes componentes: 
I. Marco epistemológico; II. Objetivo.; III. Contexto social en el que se inserta el sistema.; IV. Representación gráfica.; V. Formas de instrumentación.; VI. Competencias.

\section{A continuación se muestra cada componente.}

\section{Marco epistemológico.}

Dentro de los presupuestos que sirven de base epistemológica al Modelo de Competencias Profesionales Específicas para la formación de los especialistas en Medicina General Integral que se estructura está su definición ofrecida con anterioridad, identificada con:

$>\mathrm{El}$ proceso pedagógico profesional permanente que comienza en la formación básica y continúa en la especializada, que provoca un cambio continuo en todos los niveles de desempeño profesional y humano.

$>$ La eficiencia en la adquisición y/o desarrollo de las competencias específicas exigidas por el modelo profesional y donde se manifiestan las dos dimensiones: la técnica profesional y la humana.

> La elevación de los conocimientos científicos en una rama del saber, así como las habilidades y valores vinculada con estos.

En la base epistemológica del Modelo de Competencias para la formación que se propone se asume las funciones identificadas por Pedro Valiente y Rosa Álvarez ${ }^{(25)}$ relacionadas con las competencias educativas, estas son:

Función Informativa: Las competencias tiene como objetivo principal la producción de información caracterizadora y valorativa sobre el objeto, para la toma de decisiones con vistas a la mejora de la gestión en relación con el mismo. Esta función se explica, además, por el papel que la evaluación de las competencias debe jugar para la rendición de cuentas, a los diferentes actores sociales interesados, acerca de lo qué está ocurriendo en la práctica educativa.

Función de Control: El proceso de formación de competencias permite, tomando como base la información que produce, la verificación y constatación del cumplimento de las metas y objetivos planeados, detectar las desviaciones ocurridas, identificar lo que puede ser destacable positivamente y plantear las medidas correctivas al respecto.

Función de Diagnóstico: La evaluación de las competencias debe posibilitar una caracterización del estado del funcionamiento de los objetos evaluados (el sistema educativo en su conjunto, los programas y políticas, las instituciones educativas, el desempeño docente, el aprendizaje de los alumnos), donde resulten identificados los logros, potencialidades (fortalezas internas y oportunidades en el contexto), deficiencias, desajustes, disfunciones y barreras en un momento determinado, lo que permite operar en la derivación de acciones que coadyuven al alcance de la calidad educativa. La identificación de potencialidades se convierte en un importante recurso informativo para la adecuación del pronóstico y los objetivos planificados.

Función Educativa: La formación por competencias, dado su carácter participativo y comprometedor, debe contribuir al proceso de formación de los diferentes agentes de la comunidad educativa, al informarlos y hacerlos conscientes sobre el comportamiento de las diferentes variables que intervienen para el logro de la calidad educativa y su incidencia personal en ello. Tal toma de conciencia debe contribuir al aumento de la implicación y participación individual, al mejoramiento profesional y humano del personal que conforma la comunidad educativa y a la formación y el desarrollo del colectivo.

Función Desarrolladora: Las acciones para la adquisición de competencias profesionales específicas están encaminadas a la mejora y perfeccionamiento del proceso y los resultados de la actividad educativa y al logro de niveles superiores de calidad. En tal sentido, debe coadyuvar, cada vez más, al alcance de peldaños más elevados, sobre la base de la adopción de las mejores decisiones, sustentadas en una información objetiva, confiable y relevante. Esta función resume e integra a las anteriores al estar asociada directamente al objetivo fundamental de toda competencia educativa y justifica plenamente su necesidad." (25)

Estas funciones en el plano metodológico, refuerzan la intencionalidad educativa, formativa y desarrolladora del Modelo de Competencias Profesionales Específicas que se propone y su singularidad de que con la participación y comprometimiento con el proceso de formación de los estudiantes de la residencia médica en Medicina General Integral, se logra la adquisición de competencias profesionales específicas de los mismos, elemento que debe ser evaluado dentro del proceso de formación y al cierre de cada año académico.

\section{Objetivo.}

Este componente del Modelo de Competencias Profesionales Específicas para la formación de 
los especialistas en Medicina General Integral se refiere en primer lugar a la misión que tiene, expresado como: Valoración de la organización académica de los programas de especialidades a partir de la formación por competencias profesionales específicas y el mejoramiento del desempeño profesional de los estudiantes de postgrado que favorece la integración entre la Universidad y la Sociedad, desde una concepción desarrolladora y humanista.

Los objetivos generales que persigue este Modelo de Competencias Profesionales Específicas son:

- Identificar las deficiencias que posee la formación de postgrado en respuesta a las políticas de desarrollo de las competencias profesionales específicas en los procesos de formación del especialista de Medicina General Integral.

- Implementar las competencias profesionales específicas para la formación del especialista de Medicina General Integral, que involucre al individuo de forma activa en la solución de los problemas y en el desarrollo de sus potencialidades.

- Valorar de forma sistemática el proceso de formación académica, laboral e investigativa, en correspondencia con el modelo del egresado.

- Elevar la calidad de la atención médica hacia la comunidad desde la calidad en el proceso de formación de los residentes en Medicina General Integral.

\section{Contexto social en el que se inserta el sistema}

La Facultad de Ciencias Médicas "Dr. Enrique Cabrera Cosío" en el municipio Boyeros, en La Habana, anexado a la Universidad de Ciencias Médicas de la Habana, sirve de escenario a esta investigación, a partir de dirigir su atención a la valoración del mejoramiento del desempeño profesional de los residentes en formación.

Para la construcción del referido Modelo de Competencias Profesionales Específicas, la autora relaciona las funciones extraídas del programa de la residencia con las áreas del conocimiento y las áreas de actuación de su desempeño profesional con las que se vinculan, desde este estudio se erigen las vías para evaluar la formación por competencias profesionales específicas de los especialistas en Medicina General Integral.

Uno de las características del Modelo de Competencias Profesionales Específicas reside en que debe permitir que a través del desarrollo de las competencias profesionales específicas, los estudiantes y docentes encuentren las dificultades, necesidades y problemas a resolver en la formación, así como las potencialidades a desarrollar durante esta especialización, esto le imprime una cualidad nueva: la participación activa del residente y constituye un resultado práctico para la elevación de la calidad de los servicios de salud que son atendidos por los residentes en Medicina General Integral..

Estas relaciones se manifiestan tanto en la Etapa de elaboración, aplicación y análisis de los resultados, como en la Etapa de toma de decisiones y socialización de estos, para que posibilite la retroalimentación del proceso de adquisición de competencias profesionales específicas de los residentes en Medicina General Integral, su evaluación y la elevación de la calidad de los servicios profesionales que estos realizan en la atención en salud.

Desde las funciones extraídas del modelo del egresado la autora establece las competencias profesionales específicas, relacionadas con las áreas del conocimiento, las habilidades, el modo de actuación y los valores que logran con su desempeño profesional los médicos que cursan la especialidad en Medicina General Integral.

Este modelo de formación por competencias se acompaña con el sistema de valores que son comunes para todo el sistema de competencias y que están en correspondencia con el modelo del profesional de la especialidad de Medicina General Integral y con las exigencias sociales que se realiza a la formación permanente y continuada de los profesionales de la salud en Cuba, este sistema de valores es el siguiente:

Humanismo, Solidaridad, Altruismo, Ética, Honestidad, Austeridad, Integralidad moral y política, Patriotismo, Internacionalismo, Responsabilidad, Modestia, Sencillez, Sensibilidad, Abnegación, Sacrificio, Combatividad. 


\section{Representación gráfica}



\section{Formas de instrumentación.}

A partir del estudio del sistema de competencias del actual programa de residencia en Medicina General Integral, la autora identificó las siguientes competencias profesionales específicas que posibilitan mejoría en su desempeño profesional:

Competencia Comunicativa, entendida como el sistema de conocimientos, habilidades, actitudes y valores para la comunicación adecuada con el individuo, la familia, la comunidad y otros profesionales de la salud que garantice la obtención y transmisión de la información necesaria para la atención médica integral en un área o servicio, para la toma de decisiones, solución de problemas de salud, desde el método clínico, epidemiológico y social en el contexto de la relación individuo, familia y comunidad.

Competencia Clínica, entendida como el sistema de conocimientos, habilidades, actitudes y valores para la actuación en la atención médica en un área o servicio, para la toma de decisiones, solución de problemas de salud, desde el método clínico en el contexto de la relación individuo, familia y comunidad.

Competencia Epidemiológica, entendida como el sistema de conocimientos, habilidades, actitudes y valores para la actuación en la atención epidemiológica del individuo, la familia y la comunidad, en un área o servicio, para la identificación, toma de decisiones, solución de problemas epidemiológicos y de salud ambiental, desde el método epidemiológico
Competencia en Educación en Salud, entendida como el sistema de conocimientos, habilidades, actitudes y valores para la planificación, ejecución y evaluación de programas y proyectos de educación en salud en el individuo, familia y comunidad que permita el tratamiento , prevención y educación en salud desde el método clínico,epidemiológico con enfoque social.

Competencia Docente, entendida como el sistema de conocimientos, habilidades, actitudes y valores para la planificación, organización, ejecución y control de actividades docentes, con los estudiantes de Ciencias Médicas de pre y postgrado, dirigiendo el proceso formativo desde los escenarios de la educación en el trabajo.

Competencia Investigativa, entendida como el sistema de conocimientos, habilidades, actitudes y valores para la planificación y ejecución de investigaciones científicas en el campo de acción de su puesto de trabajo , participación en tareas de investigaciones vinculadas a problemas priorizados $y$ evaluación de investigaciones, artículos y publicaciones científicas de todo tipo desde el método científico,

Competencia Gerencial, entendida como el sistema de conocimientos, habilidades, actitudes y valores en la administración de salud expresada en su actuación para el diagnóstico, caracterización y evaluación de los procesos de salud - enfermedad y la eficiencia y calidad en la prestación de servicios a la población. 
Descripción de las Competencias Profesionales Específicas propuestas

\begin{tabular}{|c|c|}
\hline $\begin{array}{l}\text { Competencias } \\
\text { Profesionales } \\
\text { Específicas }\end{array}$ & Descripción \\
\hline \multirow{5}{*}{$\begin{array}{l}\text { Competencia } \\
\text { Comunicativa }\end{array}$} & $\begin{array}{l}\text { Escucha con atención, obtiene y sintetiza información pertinente acerca de los problemas que } \\
\text { aquejan al enfermo, y comprende el contenido de esta información. Redacta historias clínicas y } \\
\text { otros registros médicos de forma comprensible a terceros. }\end{array}$ \\
\hline & $\begin{array}{l}\text { Se comunica de modo efectivo y claro, tanto de forma oral como escrita con los pacientes, los } \\
\text { familiares, los medios de comunicación y otros profesionales. }\end{array}$ \\
\hline & $\begin{array}{l}\text { Establece una buena comunicación interpersonal, que capacite para dirigirse con eficiencia y } \\
\text { empatía a los pacientes, a los familiares, medios de comunicación y otros profesionales. }\end{array}$ \\
\hline & $\begin{array}{l}\text { Reconoce los elementos esenciales de la profesión médica, incluyendo los principios éticos y } \\
\text { las responsabilidades legales atendiendo al beneficio del paciente, de la familia, la comunidad, } \\
\text { la sociedad y la profesión, con especial atención al secreto profesional. }\end{array}$ \\
\hline & $\begin{array}{l}\text { Respeta a la autonomía del paciente, a sus creencias y cultura, desarrollando la práctica } \\
\text { profesional con respeto a otros profesionales de la salud. }\end{array}$ \\
\hline \multirow{8}{*}{$\begin{array}{l}\text { Competencia } \\
\text { Clínica }\end{array}$} & Reconoce las bases de la conducta humana normal y sus alteraciones. \\
\hline & $\begin{array}{l}\text { Comprende y reconoce los efectos, mecanismos y manifestaciones de la enfermedad sobre la } \\
\text { estructura y función del cuerpo humano. }\end{array}$ \\
\hline & $\begin{array}{l}\text { Comprende los fundamentos de acción, indicaciones y eficacia de las intervenciones } \\
\text { terapéuticas, basándose en la evidencia científica disponible. }\end{array}$ \\
\hline & $\begin{array}{l}\text { Obtiene y elabora una historia clínica que contenga toda la información relevante. Realiza un } \\
\text { examen físico general, regional y por aparatos y una valoración mental. }\end{array}$ \\
\hline & $\begin{array}{l}\text { Elabora un diagnóstico inicial y establece una estrategia diagnóstica razonada. Reconoce y trata } \\
\text { las situaciones que ponen la vida en peligro inmediato, y aquellas otras que exigen atención } \\
\text { inmediata. }\end{array}$ \\
\hline & $\begin{array}{l}\text { Establece el diagnóstico, pronóstico y tratamiento aplicando el método clínico, epidemiológico y } \\
\text { social }\end{array}$ \\
\hline & $\begin{array}{l}\text { Indica la terapéutica más adecuada de los procesos agudos y crónicos más prevalentes, así } \\
\text { como de los enfermos en fase terminal. }\end{array}$ \\
\hline & $\begin{array}{l}\text { Conocer, valora críticamente y sabe utiliza las fuentes de información clínica y biomédica para } \\
\text { obtener, organizar, interpretar y comunicar la información científica y sanitaria. }\end{array}$ \\
\hline \multirow{5}{*}{$\begin{array}{l}\text { Competencia } \\
\text { Epidemiológica }\end{array}$} & $\begin{array}{l}\text { Comprende y reconoce los agentes causantes y factores de riesgo que determinan los estados } \\
\text { de salud y el desarrollo de la enfermedad, así como los efectos del crecimiento, el desarrollo y } \\
\text { el envejecimiento sobre el individuo y su entorno social. }\end{array}$ \\
\hline & Plantea y propone las medidas preventivas adecuadas a cada situación clínica. \\
\hline & $\begin{array}{l}\text { Reconoce los determinantes de la salud en la población, tanto los genéticos como los } \\
\text { dependientes de los estilos de vida, demográficos, ambientales, sociales, económicos, } \\
\text { psicológicos y culturales. }\end{array}$ \\
\hline & $\begin{array}{l}\text { Asume su papel en las acciones de prevención y protección ante enfermedades, lesiones o } \\
\text { accidentes y mantenimiento y promoción de la salud, tanto a nivel individual como comunitario. }\end{array}$ \\
\hline & $\begin{array}{l}\text { Obtiene y utiliza datos epidemiológicos y valora tendencias y riesgos para la toma de decisiones } \\
\text { sobre salud. Realiza un Análisis de la Situación de Salud familiar e interviene oportunamente } \\
\text { ante factores ambientales, biológicos, económicos y sociales que pudieran influir de manera } \\
\text { negativa en el estado de salud de alguno de sus miembros. }\end{array}$ \\
\hline \multirow{2}{*}{$\begin{array}{l}\text { Competencia en } \\
\text { Educación en } \\
\text { Salud }\end{array}$} & $\begin{array}{l}\text { Realiza atención dispensarizada con enfoque biopsicosocial y con carácter proactivo a personas } \\
\text { y familias a través de acciones de promoción, prevención, restauración de salud y rehabilitación. }\end{array}$ \\
\hline & $\begin{array}{l}\text { Reconoce su papel en equipos multiprofesionales, asumiendo el liderazgo cuando sea } \\
\text { apropiado, tanto para el suministro de cuidados de la salud, como en las intervenciones para la } \\
\text { promoción de la salud. }\end{array}$ \\
\hline $\begin{array}{l}\text { Competencia } \\
\text { Docente }\end{array}$ & $\begin{array}{l}\text { Transmite sus conocimientos, habilidades y valores a los estudiantes de las Carreras de } \\
\text { Ciencias Médicas y otros profesionales, en el marco de la Educación en el trabajo y otras formas } \\
\text { de enseñanza dentro del proceso docente educativo, así como, como capacita a los líderes de } \\
\text { la comunidad en que se desempeña }\end{array}$ \\
\hline \multirow{3}{*}{$\begin{array}{l}\text { Competencia } \\
\text { Investigativa }\end{array}$} & $\begin{array}{l}\text { Utiliza las tecnologías de la información y la comunicación en las actividades clínicas, } \\
\text { terapéuticas, preventivas y de investigación. }\end{array}$ \\
\hline & $\begin{array}{l}\text { Mantiene y utiliza los registros con información del paciente para su posterior análisis, } \\
\text { preservando la confidencialidad de los datos. Formula hipótesis, recolecta y valora de forma } \\
\text { crítica la información para la resolución de problemas, siguiendo el método clínico y científico. }\end{array}$ \\
\hline & $\begin{array}{l}\text { Mantiene un interés constante por la superación profesional, actualiza sus conocimientos, realiza } \\
\text { investigaciones científicas que abordan los principales problemas de salud que afecten a su } \\
\text { comunidad. }\end{array}$ \\
\hline $\begin{array}{l}\text { Competencia } \\
\text { Gerencial }\end{array}$ & $\begin{array}{l}\text { Conoce, valora y utiliza los recursos humanos y materiales existentes en la comunidad, para } \\
\text { garantizar un óptimo estado de salud, así como para enfrentar situaciones especiales } \\
\text { (desastres, tiempo de guerra y otras) }\end{array}$ \\
\hline
\end{tabular}




\section{Conclusiones}

A partir del proceso de abstracción logrado alrededor del objeto de estudio, la formación de especialistas en Medicina General Integral, se logra la elaboración del Modelo de Competencias Profesionales Específicas para la formación de los especialistas en Medicina General Integral fundamentado desde las Ciencias Pedagógicas. Se reconoce que la investigación enriquece a las Ciencias Pedagógicas, en general, y a la Teoría de la Educación Avanzada, en particular, con la identificación de regularidades que se establecen en las relaciones entre la organización académica de los programas de especialidades a partir de la adquisición y desarrollo de las competencias profesionales específicas y el desempeño profesional de los estudiantes de postgrado, que favorece la integración entre la Universidad y la Sociedad, desde una concepción desarrolladora y humanista que involucra al individuo con la formación que realiza de forma activa. Estas regularidades revelan la concreción del proceso de formación por competencias profesionales específicas desde la concepción que propone la Educación Avanzada. Esta investigación ofrece a través de sus resultados, una posible solución a los problemas que se manifiestan en el proceso de formación en las especialidades, particularmente en la especialidad de Medicina General Integral y su impacto en el desempeño profesional de los sujetos que las cursan, principal meta de la investigación, al profundizar en el proceso de formación por competencias profesionales específicas de los médicos en Cuba.

\section{Referencias}

1. Tejeda, Díaz Rafael y Sánchez del Toro, Pedro R. (2010). La formación basada en competencias en los contextos universitarios. Libro electrónico: Centro de Estudios sobre Ciencias de la Educación Superior. Universidad de Holguín. "Oscar Lucero Moya”. Pág. 4 ISBN: 978-959-160961-8 300 378. Cuba. Citado julio 2017

2. Ministerio de Educación Superior. Resolución Ministerial No. 132/2004. Reglamento de la Educación de Posgrado de la República de Cuba.

3. Ministerio de Salud Púbica. Plan de Estudios de la Residencia de MGI. 4ta. versión. La Habana: MINSAP; 2004.
4. Ramos Hernández R, Díaz Díaz AA. Propuesta de perfeccionamiento de tarjeta de evaluación de residentes de Medicina General Integral. Educación Médica Superior [revista en Internet]. 2015 [citado 2017 Oct 22]; 29(3): Disponible en: http://www.ems.sld.cu/index.php/ems/article /view/492

5. Alemañy Pérez, Eduardo J. Tesis doctoral. Universidad de Ciencias Médicas de La Habana. 2008.

6. Díaz Díaz, Alejandro A, "Modelo de Evaluación de los procesos formativos de los residentes en Medicina General Integral". Tesis doctoral. UCM. La Habana. 2012: Pág. 18.

7. Salas Perea, R.S. Los procesos formativos, la competencia profesional y el desempeño laboral en el Sistema Nacional de Salud de Cuba .Revista Cubana de Educación Médica Superior. 2012; 26 (2):163-165. Editorial Citado julio 2017

8. Pérez Á $M$, Rodríguez R. Pedagogía, Andragogía, educación de jóvenes y adultos. Paradigmas educativos alternativos. Material didáctico para la Maestría en Ciencias de la Educación. Mención en Educación de Adultos.: Editorial Pueblo y Educación. La Habana, Cuba. 2006. Pág 6.

9. Añorga, J, Che, J, Colado, J (et al). La parametrización en la investigación educativa. Revista Varona No. 43. La Habana. 2014. Pág. Alpízar Caballero, L.B. Tesis doctoral. Universidad de Ciencias Médicas de La Habana. 2007.Citado julio 2017

10.Ministerio de Salud Púbica. Plan de Estudios de la Residencia de MGl. 4ta. versión. La Habana: MINSAP; 2004.

11.Bringas J A. Modelo de planificación estratégica universitaria. [Tesis en opción al Grado Científico de Doctor en Ciencias Pedagógicas]: Instituto Superior Pedagógico "Enrique José Varona"; La Habana, Cuba 1999.

12. Blanco A. Introducción a la Sociología de la Educación: Editorial Pueblo y Educación; La Habana, Cuba 2002

13.Añorga, J (et al). La teoría alternativa: Educación Avanzada; fundamentos teóricos - prácticos de los procesos de perfeccionamiento de los Recursos Humanos. Material impreso. ISPEJV. Ciudad Habana, Cuba; 2014. 
14.Freyre P. Pedagogía del oprimido. Buenos Aires, Argentina: Editorial Siglo XXI; 1983.

15.Pérez, Á M. Alfabetización y Postalfabetización. Género y Ruralidad. Curso Pre-reunión No. 2. Seminario Internacional sobre Políticas y Programas de Alfabetización y Postalfabetización. Editorial Pueblo y Educación. La Habana, Cuba. 2006.

16. Ferrer M T. Las Habilidades pedagógico profesionales de los maestros primarios. Tesis en opción al Grado Científico de Doctor en Ciencias Pedagógicas. Instituto Superior Pedagógico "Enrique José Varona"; La Habana, Cuba. 2002.

17. Pérez, F. Programa Educativo para la superación de los docentes de la Educación de Jóvenes y Adultos. Tesis doctoral. UCP. "EJ. Varona". La Habana. 2010.

18. Torres, P. Para que la evaluación educativa "sirva para algo": la mejora escolar. Revista. El evaluador educativo. Año II. No.1. La Habana. 2012.

19. Martín, R. Modelo Pedagógico para la Evaluación Institucional de Centros Politécnicos. Tesis doctoral. La Habana, Cuba: Instituto Superior Pedagógico "Enrique José Varona"; 2009.

20.Moreno, M. Propuesta metodológica para evaluar la eficacia del proceso pedagógico áulico en la Secundaria Básica. Tesis en opción del grado de Doctor en Ciencias Pedagógicas. Instituto Superior Pedagógico "Rafael María de Mendive". Pinar del Río. 2005.

21.Valiente, P. y M. Guerra. Evaluación de Sistemas, Programas y Centros Educativos. (Material Básico de la Maestría en Supervisión Educativa).IPLAC. La Habana. 2008.

22.Añorga, J; Valcárcel, N; Pérez García AM. Modelo de las competencias en profesionales en ejercicio La Habana, Cuba: Instituto Superior Pedagógico "Enrique José Varona"; 2014.

23.Terrero, A. Modelo pedagógico para la alfabetización por radio. Tesis en opción al Grado Científico de Doctor en Ciencias Pedagógicas. Instituto Pedagógico Latinoamericano y Caribeño; La Habana, Cuba. 2006.

24.De Armas, N. Formación vocacional. Revista Educación. Editorial Pueblo y Educación La Habana. 1985.

25.Valiente, P.; Álvarez, R: "Metodología para evaluar el impacto de la superación de directivos educacionales". Revista Digital UMBRAL 200 - No. 15 Disponible en www.reduc.cl. 2004. 